\title{
Cardioplegia cristalóide, barotrauma e função endotelial: considerações experimentais
}

\author{
Paulo Roberto B. ÉVORA*, Paul J. PEARSON**, Marilyn OELTJEN**, Berent DISCIGIL**, \\ Hartzell V. SCHAFF ${ }^{\star \star}$
}

RBCCV 44205-298

\begin{abstract}
Évora PR B, Pearson P J, Oeltjen M, Discigil B, Schaff H V - Cardioplegia cristalóide, barotrauma e funçāo endotelial: consideraçōes experimentais. Rev Bras Cir Cardiovasc 1996; 11 (2): 115-22.

RESUMO: O presente ensaio experimental estudou o efeito da infusão de solução cardioplégica cristalóide a altas pressōes sobre a função endotelial de artérias epicárdicas de cães. Não se encontraram alterações a nível de receptores (curvas dose-respostas à ACH e ADP; da transduçāo do sinal iniciado nos receptores/sitema de G-proteínas (fluoreto de sódio) e nos processos intracelulares da produção de EDRF/ NO (fosfolipase C e ionóforo do cálcio A23187). A função da musculatura lisa vascular não foi afetada quando se analisaram as respostas relaxantes (nitroprussiato de sódio e isoproterenol) e contráteis ( $\mathrm{KCl}$ e prostaglandina 2alfa). Estes achados permitem as seguintes considerraçōes especulativas: a) O barotrauma produzido pela infusão da cardioplegia cristalóide a altas pressōes ocorreria apenas em circulações coronarianas previamente doentes? b) Uma vez que as infusōes duraram de 2 a 3 minutos, seria o barotrauma coronariano um fenômeno dependente do tempo de infusão? c) Para que ocorra o barotrauma seriam necessários níveis mais elevados de potássio? d) Questionar a existência do fenômeno do barotrauma coronariano produzido pela infusão de soluçōes cadioplégicas pelo menos nas condiçōes experimentais utilizadas. e) A metodologia empregada estuda apenas as reatividades vasculares de artérias coronarias epicárdicas. Estas artérias seriam menos sensíveis aos efeitos da pressào de infusāo da cardioplegia do que a microcirculaçāo coronariana? f) Seria a circulação coronária do cão menos sensivel a altas pressōes do que do homem? Estas observaçōes experimentais sugerem quę a infusão de cardioplegia cristalóide, moderadamente hipocalêmica, a altas pressōe em um tempo de 2 a 3 minutos, não interfere com a produçāo de EDRF/NO pelo endotélio de coronárias epicárdicas do cão.
\end{abstract}

DESCRITORES: Parada cardiaca induzida, métodos. Barotrauma. Endotélio vascular, efeito de drogas. Pericárdio, efeito de drogas. Vasos coronários, efeito de drogas. Soluçōes cardioplégicas, farinacologia.

INTRODUÇĀO

Vários grupos têm demonstrado uma profunda e generalizada evidência histológica de lesão endotelial após infusão de cardioplegia cristalóide ${ }^{(1-3)}$, mas os resultados destes trabalhos podem ser atribuídos aos efeitos da pressão e shear stress no endotélio vascular. Altas pressões de infusão podem lesar o endotélio coronariano e de artérias cerebrais $(2,4)$. Deve-se lembrar que o contrôle da pressão de in-

Trabalho realizado em:Cardiac Surgical Research and Section of Cardiovascular Surgery. Mayo Clinic and Mayo Foundation. Rochester, Minnezota, USA, e no Departamento de Cirurgia, Ortopedia e Traumatologia da Faculdade de Medicina de Ribeirăo Preto da Universidade de Săo Paulo. Ribeirāo Preto, SP, Brasil.

Recebido para publicação em 2 de abril de 1996.

* Da Sessão de Pós-Graduação do Departamento de Cirurgia, Ortopedia e Traumatologia da Faculdade de Medicina de Ribeirão Preto. Fellow da Mayo Clinic à época da elaboração deste trabalho.

** Do Cardiac Surgical Research and Section of Cardiovascular Surgery, Mayo Clinic.

Endereço para correspondência: Paulo Roberto B. Evora. Rua Rui Barbosa, 455, Apto. 140, CEP:14015-120 - Tel. (016) 636.4149 e 634.0992. 
fusão não garante shear stress normal nas artérias coronarianas epicárdicas. Mas, parece provável que a lesão da célula endotelial pela infusão de cardioplegia é, ao menos em parte, uma expressão da lesão induzida por shear stress e barotrauma. Esta hipótese é consistente com o achado de que fluidos com diferentes viscosidades induzem diferentes gráus de lesão endotelial durante a infusão coronariana ${ }^{(2)}$. No presente trabalho estudou-se o efeito da infusão de solução cardioplégica cristalóide a altas pressões sobre a função endotelial de artérias coronarianas epicárdicas de cães.

\section{MATERIAL E MÉTODOS.}

Cães mestiços $(n=6)$ pesando entre $25 \mathrm{~kg}$ e 30 $\mathrm{kg}$ foram anestesiados e ventilados com auxílio de respirador. Através de uma toracotomia lateral direita, as veias cavas foram enlaçadas e ocluídas. A aorta foi, então, pinçada, procedendo-se à infusão de cardioplegia cristalóide hipotérmica $\left(7^{\circ} \mathrm{C}\right)$ através de uma cânula na aorta ascendente a um fluxo tal que mantinha a pressão aórtica proximal entre 95 e $190 \mathrm{mmHg}(136,66 \pm 39,32 \mathrm{mmHg})$ (Figura 1). Após esta infusão, o coração foi excisado, procedendo-se ao preparo das artérias coronárias para o estudo em organ chambers (Figuras 2 e 3 ). Utiliza-

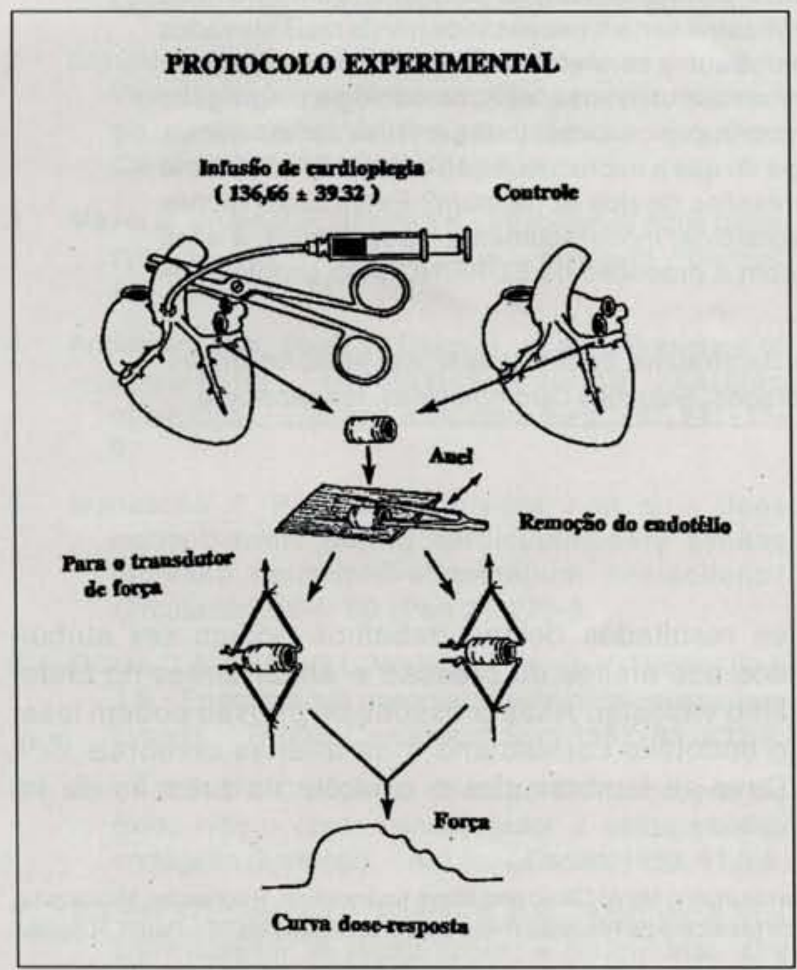

Fig. 1 - Protocolo experimental utilizado para o estudo in vitro do efeito da infusão de solução cardioplégica com altas pressōes.

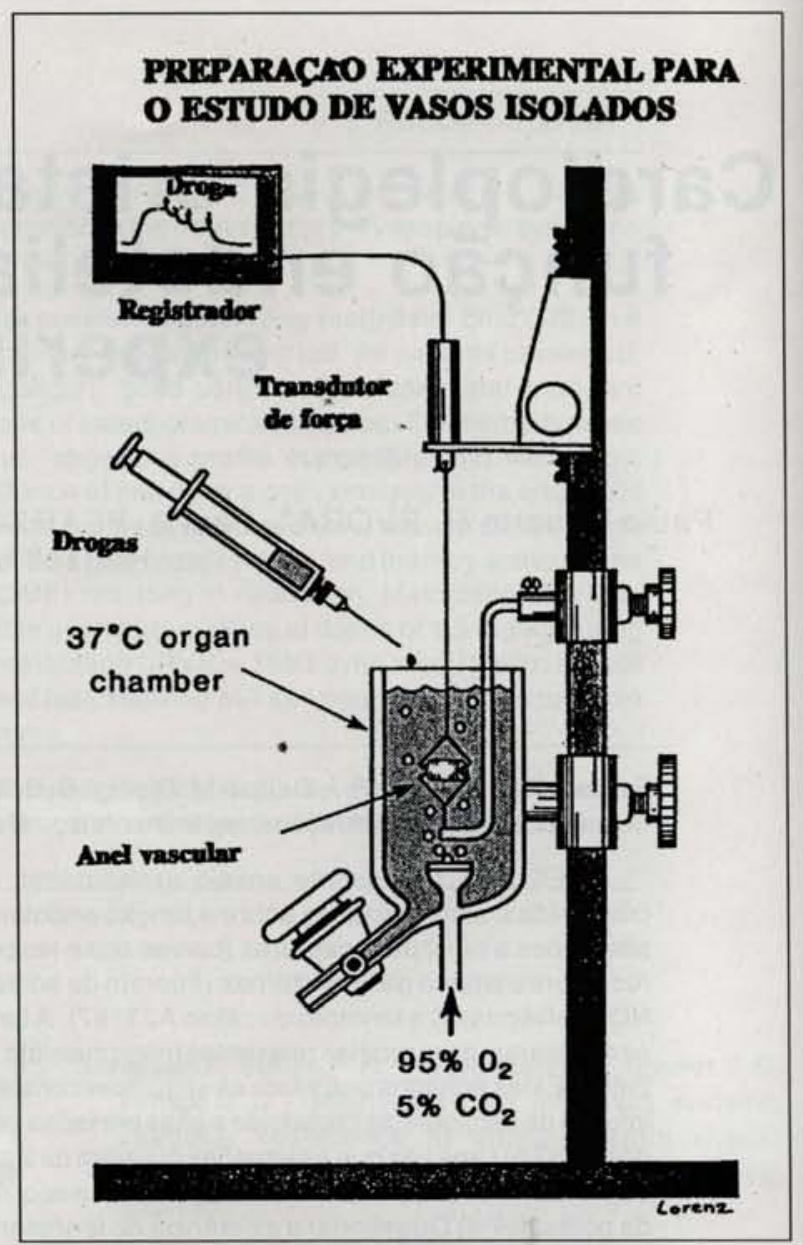

Fig. 2 - Representação esquemática do sistema de organ chambers uitlizado para o estudo farmaciológico in vitro da função endotelial.

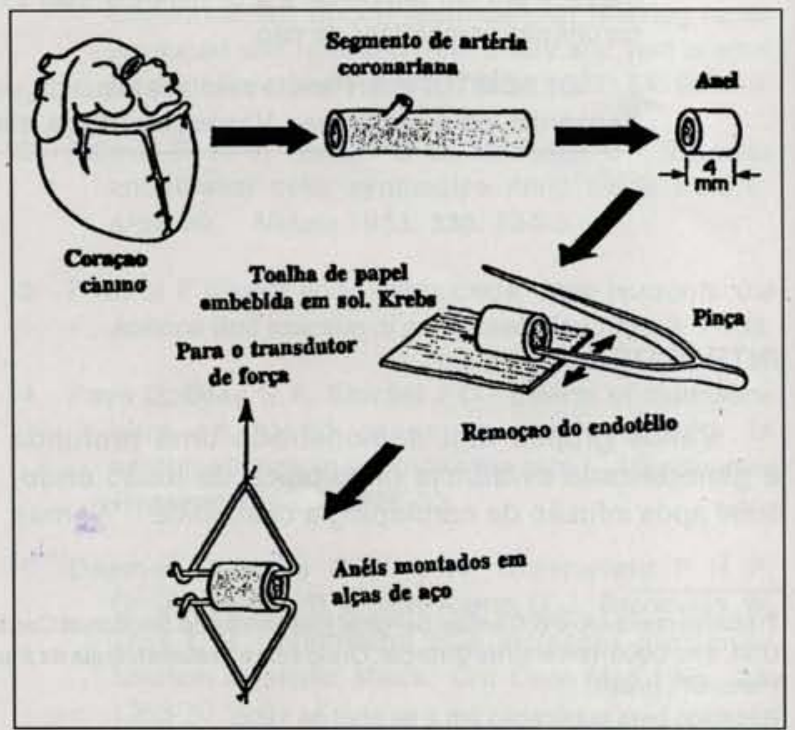

Fig. 3 - Representação esquemática do método manual utilizado para a remoção mecânica do endotélio vascular. 
Évora PRB, Pearson PJ, Oeltjen M, Discigil B, Schaff HV - Cardioplegia cristalóide, barotrauma e função endotelial: consideraçōes experimentais. Rev Bras Cir Cardiovasc 1996; 11 (2): 115-22.

ram-se as seguintes drogas: acetilcolina cloridrato, adenosina difosfato (ADP), indometacina, fluoreto de sódio, fosfolipase $C$, prostaglandina $F_{2}$, nitroprussiato de sódio e $( \pm)$ cloridrato de isoproterenol (todas da Sigma Chemical Company, St. Louis, Missouri). Preparou-se, diariamente, uma solução cardioplégica comercial (Plegisol, Abbott Laboratories, North Chicago, Illinois), adicionando-se $10 \mathrm{ml}$ de bicarbonato de sódio $8,4 \%$ a $1000 \mathrm{ml}$ da solução cardioplégica, obtendo-se a seguinte composição final ( $\mathrm{mEq} / \mathrm{l})$ : cálcio 2.4, magnésio 3.2 , potássio 16 , sódio 120 , e cloro 160 . As soluções foram mantidas a $7^{\circ} \mathrm{C}$ até o momento de seu uso. Como controle realizaram-se curvas dose-respostas aos diversos agonistas da produção do óxido nítrico em coronárias de cães $(n=6)$ não submetidos a infusão de solução cardioplégica.

\section{RESULTADOS.}

Os estudos comparativos das curvas dose-respostas entre segmentos de artérias coronárias submetidas a infusão de cardioplegia cristalóide e segmentos coronarianos controles permitiram as seguintes observações:

a) Não se encontraram alterações a nível de receptores: as curvas doses-respostas à $\mathrm{ACH}$ e ao ADP foram normais não diferindo estatisticamente dos resultados obtidos em artérias coronárias controles (Gráficos 1 e 2 ).

GRÁFICO 1

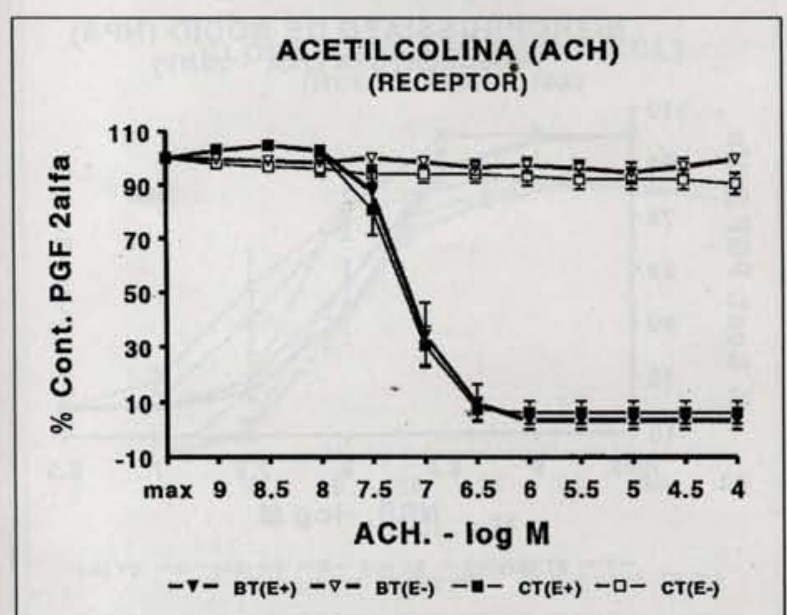

Curvas dose-respostas à acetilcolina ( $\mathrm{ACH}$ ) em segmentos arteriais controles e submetidos à infusão de cardioplegia com altas pressões $(N=5)$. Os anéis foram primeiro contraidos com prostaglandina 2alfa $e$ expostos a concentraçōes crescentes de $\mathrm{ACH}$. Apresentam-se os resultados sob a forma de média e desvio-padrão. (Diferenças estatisticamente não significantes - ANOVA, $P<0,05)$. BT $(E+)=A$ nel coronariano com endotélio submetido à infusão pressórica; $B T(E-)=$ Anel coronariano submetido à infusão pressórica sem endotélio; CT $(E+)=$ Anel coronariano controle com endotélio; $C T(E-)=$ Anel coronariano controle sem endotélio.

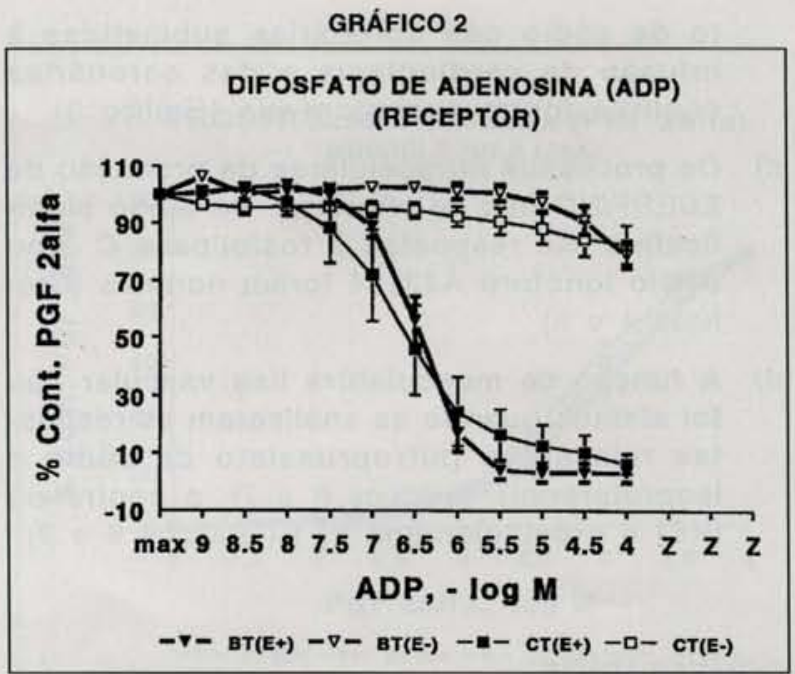

Curvas dose-respostas ao difosfato de adenosina (ADP) em segmentos arteriais controles e submetidos à infusâo de cardioplegia com altas pressōes $(\mathrm{N}=5)$. Os anéis foram primeiro contraidos com prostaglandina 2 alfa e expostos a concentrações crescentes de ADP. Apresentam-se os resultados sob a forma de média e desvio-padrāo. (Diferenças estatisticamente nāo significantes - ANOVA, $P<0,05$ ). BT $(E+)=$ Anel coronariano com endotélio submetido à infusăo pressórica; BT (E-) = Anel coronariano submetido à infusão pressórica sem endotélio; $C T(E+)=$ Anel coronariano controle com endotélio; $C T(E-)=$ Anel coronariano controle sem endotélio.

b) Não se encontraram alteraçōes a nível do sistema de transdução do sinal, iniciado nos receptores, para os processos mobilizadores do cálcio intracelular. As respostas ao fluore-

GRÁFICO 3

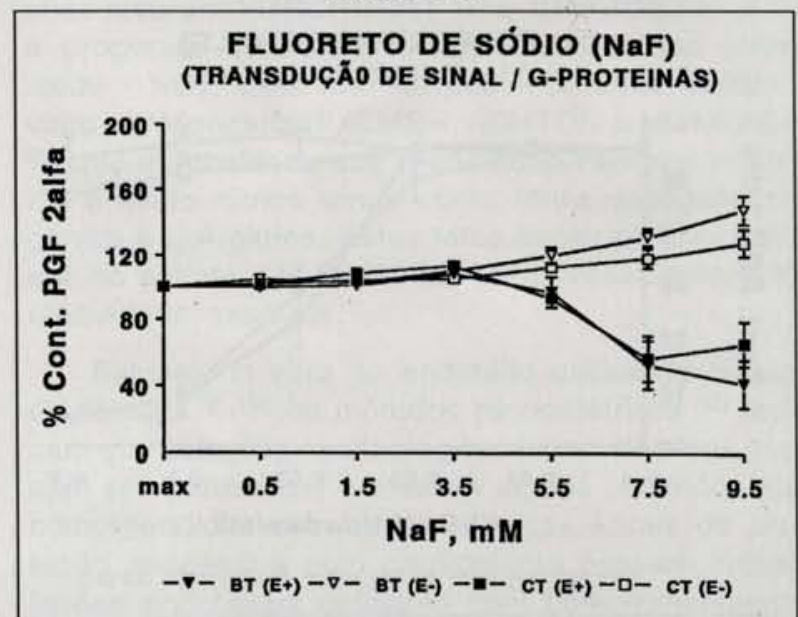

Curvas dose-respostas ao fluoreto de sódio (NaF) em segmentos arteriais controles e submetidos à infusão de cardioplegia com altas pressōes $(\mathrm{N}=5)$. Os anéis foram primeiro contraídos com prostaglandina 2alfa e expostos a concentraçōes crescentes de NaF. Apresentam-se os resultados sob a forma de média e desvio-padrão. (Diferenças estatisticamente não significantes - ANOVA, $P<0,05)$. BT $(E+)=A n e l$ coronariano com endotélio submetido à infusão pressórica; $\mathrm{BT}(\mathrm{E}-\mathrm{-})=$ Anel coronariano submetido à infusão pressórica sem endotélio; CT $(E+)=$ Anel coronariano controle com endotélio; $C T(E-)=$ Anel coronariano controle sem endotélio. 
Évora PRB, Pearson PJ, Oeltjen M, Discigil B, Schaff HV - Cardioplegia cristalóide, barotrauma e funçāo endotelial: consideraçōes experimentais. Rev Bras Cir Cardiovasc 1996; 11 (2): 115-22.

to de sódio das coronárias submetidas à infusão de cardioplegia e das coronárias controle foram superponíveis (Gráfico 3 ).

c) Os processos intracelulares da produção de EDERF/NO não se alteraram de modo significativo. As respostas à fosfolipase $\mathrm{C}$ e ao cálcio ionóforo A23184 foram normais (Gráficos 4 e 5)

d) A função da musculatura lisa vascular não foi afetada quando se analisaram as respostas relaxantes (nitroprussiato de sódio e isoproterenol) (Gráficos 6 e 7) e contráteis ( $\mathrm{KCl}$ e prostaglandina $\mathrm{F}_{2}$ ) (Gráficos 8 e 9 ).

\section{COMENTÁRIOS}

Em 1980, FURCHGOTT \& ZAWADSKI ${ }^{(5)}$ estabeleceram a presença do endotélio como condição para um vaso sangüíneo relaxar sob a ação da acetilcolina. A interpretação deste fenômeno biológico levou à proposição de que o endotélio produziria um fator relaxante, o qual foi denominado de fator relaxante derivado do endotélio (EDRF endothelium-derived relaxing factor). Durante cinco anos estabeleceu-se apenas que este fator não era um prostanóide e era difusível. A partir de 1985 , com o início dos métodos de culturas de células

GRÁFICO 4

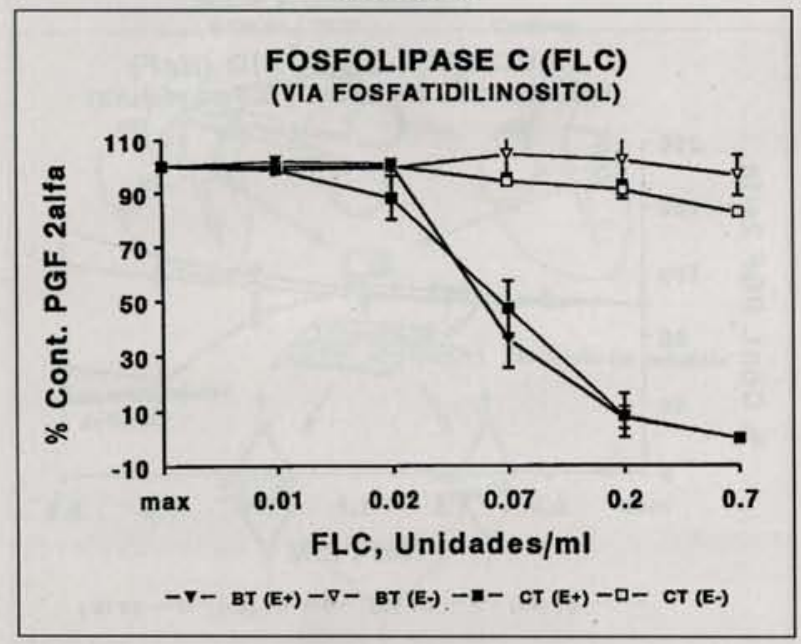

Curvas dose-respostas à fosfolipase C (FLC) em segmentos arteriais controles e submetidos à infusão de cardioplegia com altas pressões $(\mathrm{N}=5)$. Os anéis foram primeiro contraidos com prostaglandina 2alfa $e$ expostos a concentraçōes crescentes de FLC. Apresentam-se os resultados sob a forma de média e desvio-padrāo. (Diferenças estatisticamente não significantes - ANOVA, $\mathrm{P}<0,05)$. BT $(E+)=$ Anel coronariano com endotélio submetido à infusão pressórica; $B T(E-)=$ Anel coronariano submetido à infusão pressórica sem endotélio; CT $(E+)=$ Anel coronariano controle com endotélio; $C T(E-)=$ Anel coronariano controle sem endotélio.

\section{GRÁFICO 5}

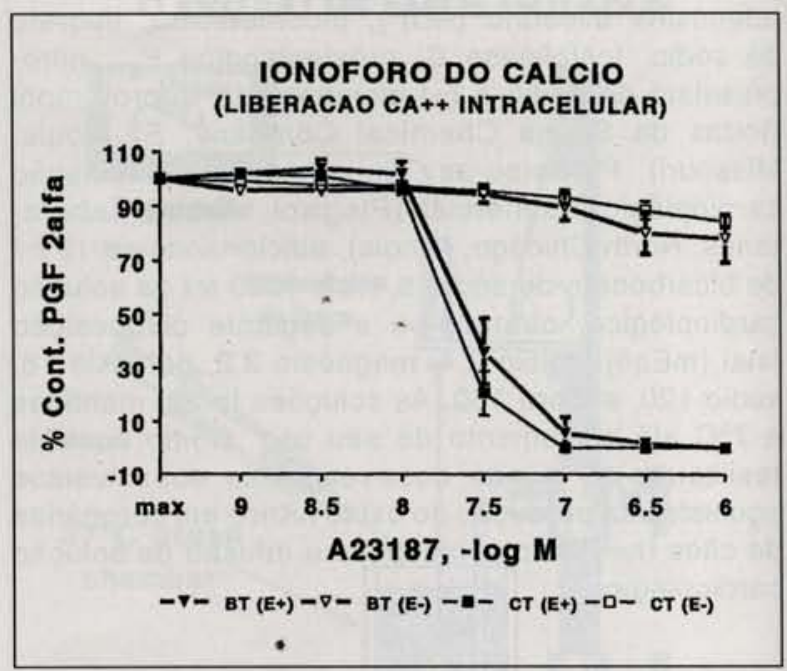

Curvas dose-respostas ao ionóforo do cálcio (A23187) em segmentos arteriais controles e submetidos à infusão de cardioplegia com altas pressōes $(\mathrm{N}=5)$. Os anéis foram primeiro contraídos com prostaglandina 2alfa e expostos a concentraçōes crescentes de A23187. Apresentam-se os resultados sob a forma de média e desvio-padrão. (Diferenças estatisticamente não significantes - ANOVA, $P<0,05)$. BT $(E+)=$ Anel coronariano com endotélio submetido à infusão pressórica; BT $(\mathrm{E}-)=$ Anel coronariano submetido à infusāo pressórica sem endotélio; $\mathrm{CT}(\mathrm{E}+)=$ Anel coronariano controle com endotélio; $\mathrm{CT}(\mathrm{E}-)=$ Anel coronariano controle sem endotélio.

GRÁFICO 6

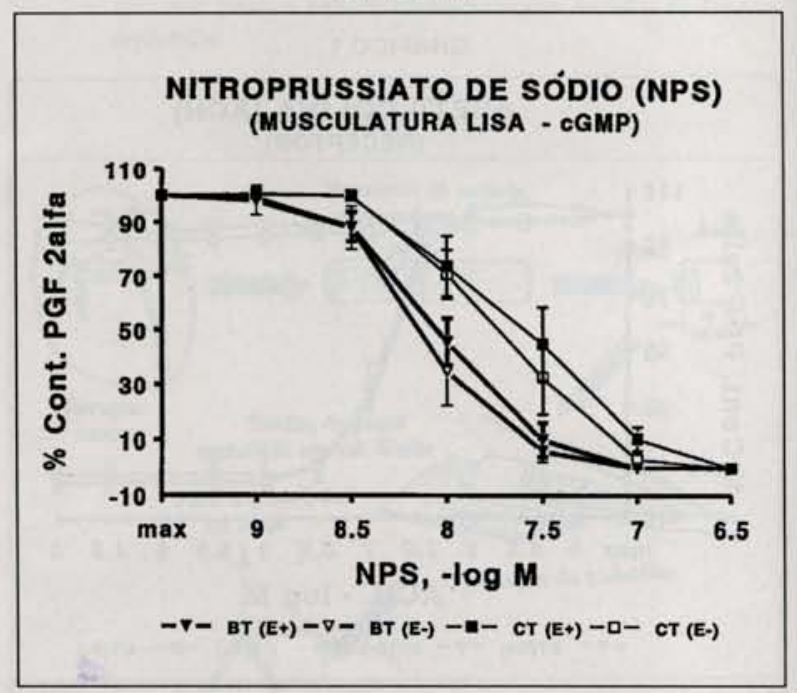

Curvas dose-respostas ao nitroprussiato de sódio (NPS) em segmentos arteriais controles e submetidos à infusão de cardioplegia com altas pressōes $(\mathrm{N}=5)$. Os anéis foram primeiro contraidos com prostaglandina 2alfa e expostos a concentrações crescentes de NPS. Apresentam-se os resultados sob a forma de média e desvio-padrão. (Diferenças estatisticamente não significantes - ANOVA, P<0,05). BT $(E+)=$ Anel coronariano com endotélio submetido à infusão pressórica; $\mathrm{BT}(\mathrm{E}-\mathrm{-}=$ = Anel coronariano submetido à infusão pressórica sem endotélio; $C T(E+)=$ Anel coronariano controle com endotélio; $C T(E-)=$ Anel coronariano controle sem endotélio. 
Évora PRB, Pearson P J, Oeltjen M, Discigil B, Schaff HV - Cardioplegia cristalóide, barotrauma e função endotelial: consideraçōes experimentais. Rev Bras Cir Cardiovasc 1996;11 (2): 115-22.

GRÁFICO 7

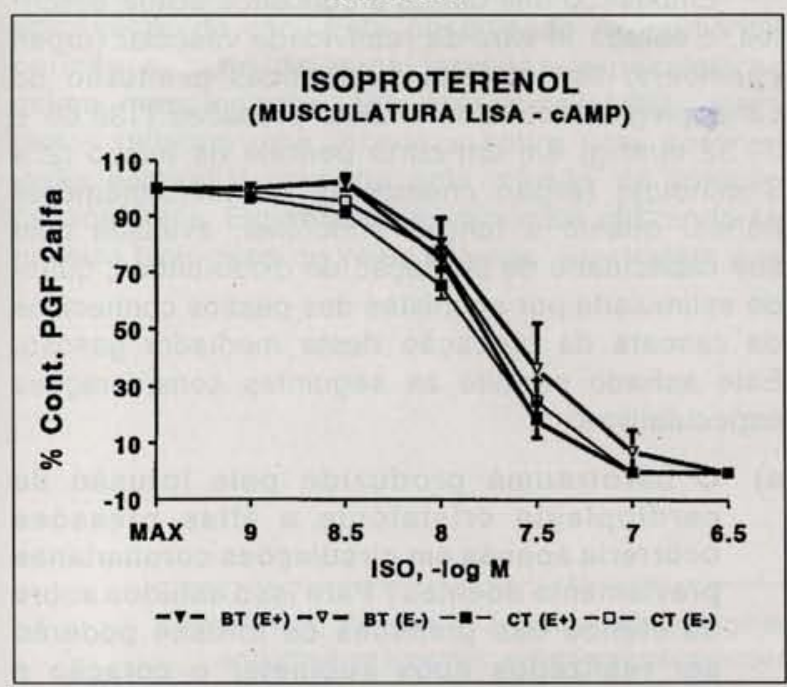

Curvas dose-respostas ao isoproterenol (ISO) em segmentos arteriais controles e submetidos à infusão de cardioplegia com altas pressões $(\mathrm{N}=5)$. Os anéis foram primeiro contraídos com prostaglandina 2alfa e expostos a concentraçōes crescentes de ISO. Apresentam-se os resultados sob a forma de média e desvio-padrão. (Diferenças estatisticamente nāo significantes - ANOVA, $P<0,05)$. BT $(E+)=A n e l$ coronariano com endotélio submetido à infusão pressórica; $B T(E-)=$ Anel coronariano submetido à infusāo pressórica sem endotélio; CT $(E+)=$ Anel coronariano controle com endotélio; CT $(E-)=A n e$ coronariano controle sem endotélio.

\section{GRÁFICO 8}

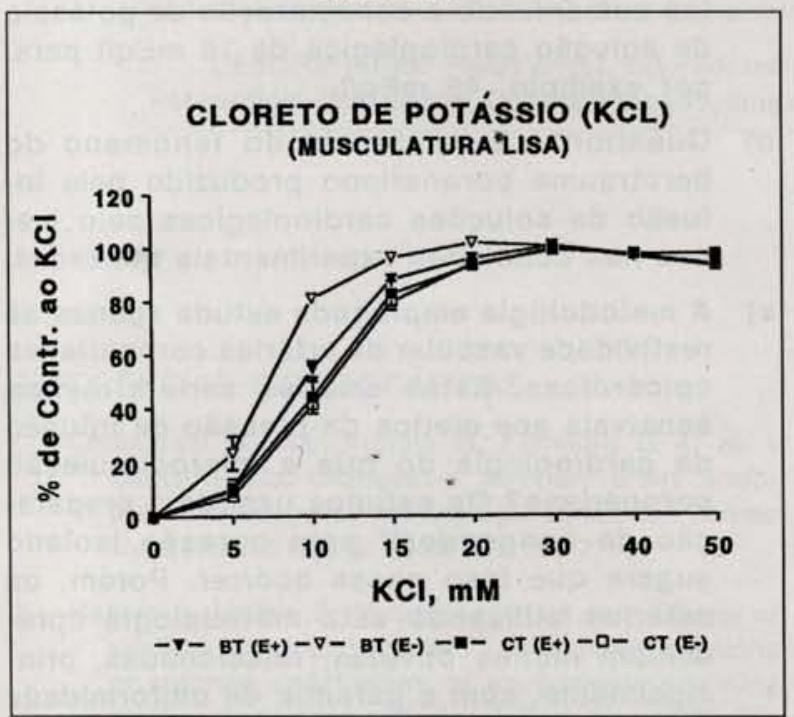

Curvas dose-respostas ao clorêto de potássio $(\mathrm{KCl})$ em segmentos arteriais controles e submetidos à infusão de cardioplegia com altas pressōes $(\mathrm{N}=5)$. Os anéis foram crescentes de $\mathrm{KCl}$. Apresentam-se os resultados sob a forma de média e desvio-padrăo. (Diferenças estatisticamente não significantes - ANOVA, $P<0,05)$. BT $(E+)=$ Anel coronariano com endotélio submetido à infusão pressórica; $\mathrm{BT}(\mathrm{E}-\mathrm{-})=$ Anel coronariano submetido à infusão pressórica sem endotélio; CT $(E+)=$ Anel coronariano controle com endotélio; $\mathrm{CT}(\mathrm{E}-)=$ Anel coronariano controle sem endotélio.

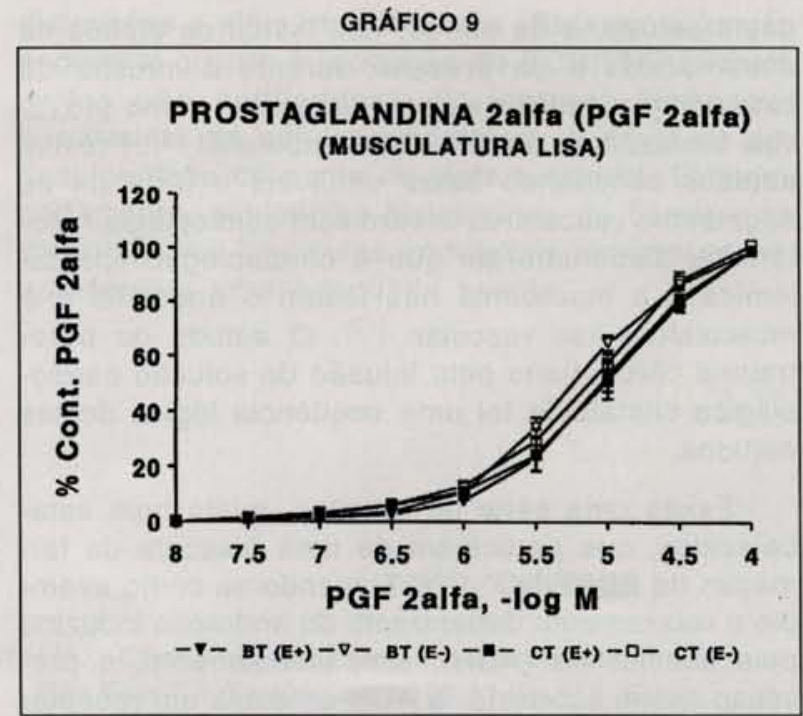

Curvas dose-respostas prostaglandina-2alfa (PGF2alfa) em segmentos arteriais controles e submetidos à infusão de cardioplegia com altas pressōes $(\mathrm{N}=5)$. Os anéis foram expostos a concentraçōes crescentes de PGF2alfa. Apresentam-se os resultados sob a forma de média e desvio-padrão. (Diferenças estatisticamente não significantes - ANOVA, $P<0,05)$. BT $(E+)=$ Anel coronariano com endotélio submetido à infusão pressórica; BT (E-) = Anel coronariano submetido à infusão pressórica sem endotélio; $\mathrm{CT}(\mathrm{E}+)=$ Anel coronariano controle com endotélio; $\mathrm{CT}(\mathrm{E}-\mathrm{-}$ = Anel coronariano controle sem endotélio

endoteliais, foi possível a manipulação bioquímica e farmacológica deste fator relaxante, estabelecendo-se que sua ação era muito semelhante às ações dos vasodilatadores coronarianos. Estas observações levaram FURCHGOTT (6) e IGNARRO et al (7) a proporem que o EDRF era óxido nítrico (nitric oxide - NO). Este fato foi posteriormente comprovado por Moncada (PALMER, ASHTON \& MONCADA $\left.{ }^{8}\right)$, que estabeleceu que o endotélio vascular produzia o óxido nítrico tendo como fonte endógena orgânica a L-Arginina. Estes fatos iniciaram uma nova era no estudo das moléstias cardiovasculares e da reatividade vascular.

Estudos in vitro do endotélio utilizando cultura de células $(9,10)$ ou métodos morfométricos (2) indicam que soluções cardioplégicas hipercalêmicas possam ser citotóxicas, e existem alguns conceitos mal compreendidos de que as técnicas atuais de proteção miocárdica com cardioplegia possam induzir lesões endoteliais celulares com comprometimento dos mecanismos protetores contra vasoespasmo e trombose intravascular.

Usando corações isolados, SALDANHA \& HEARSE (11) encontraram que a cardioplegia hipercalêmica pode causar lesão celular endotelial. Nestes experimentos, entretanto, os corações de ratos foram perfundidos por 30 minutos com solução hipercalêmica oxigenada a $100 \mathrm{mmHg}$. O planejamento 
deste estudo, e de outros, não isolou os efeitos do shear stress e da pressão durante a infusão da cardioplegia, fatores estes conhecidos como prováveis causadores de disfunção endotelial (4). Prévios estudos eliminando estas variáveis e tratando os segmentos vasculares in vitro com cardioplegia hipotérmica demonstraram que a cardioplegia hipercalêmica e a hipotermia não lesam o endotélio e a musculatura lisa vascular (12). O estudo do barotrauma coronariano pela infusão de solução cardioplégica cristalóide foi uma seqüência lógica destes estudos.

Existe uma série de eventos, muito bem estabelecidos, que participam de uma "cascata da formação do EDRF/NO" (13). Tomando-se como exemplo o relaxamento dependente do endotélio induzido pela acetilcolina $(\mathrm{ACH})$, simplificadamente, o processo assim ocorrería: a $\mathrm{ACH}$ estimula um receptor muscarínico, libera EDRF/NO que se difunde para a musculatura lisa vascular, provocando um aumento do GMP cíclico com vasodilatação. Esta é apenas uma visão parcial, já que o processo é um pouco mais elaborado, envolvendo um sistema de transdução com mensageiros secundários que incluem G-proteínas, as quais fazem a conexão com a via fosfatidil-inositol, que por sua vez mobiliza o cálcio intracelular necessário para a formação do óxido nítrico a partir da L-arginina pela ação da óxido nítrico sintetase.

Existem marcadores" farmacológicos para o estudo dos diversos passos da via clássica de liberação do EDRF/NO, todos eles podendo ter uma participação na gênese de moléstias cardiovasculares. As alterações a nível de receptores são estudadas por diversos agonistas, sendo muito utilizados a $\mathrm{ACH}$ como um neurotransmissor estimulador de receptores muscarínicos e o ADP como um produto plaquetário. Em relação à transdução do sinal entre receptores e os processos intracelulares, embora ainda não totalmente aceito como um marcador definitivo, inúmeros experimentos sugerem que o fluoreto de sódio produz relaxamento dependente do endotélio através de uma via sensível à toxina pertussis $(14,15)$. A "ferramenta" para estudar-se a via fosfatidil inositol é a fosfolipase $C$, ressaltandose a sua especificidade, uma vez que as fosfolipases B e D não induzem vasodilatação dependente do endotélio. É possivel que o sinergismo entre o EDRF/ $\mathrm{NO}$ e a prostaciclina ocorra através da via fosfatidilinositol (16). A seguir, merece destaque o ionóforo cálcico A23187, o qual, promovendo uma vasodilatação dependente do endotélio, mostra que a célula endotelial mantém a capacidade de produzir EDRF/ NO. O estudo da função da musculatura lisa vascular estuda como "marcadores" farmacológicos o isoproterenol (AMP cíclico) e o nitroprussiato de sódio (GMP cíclico).
Embasado nos dados e conceitos acima descritos, o estudo in vitro da reatividade vascular (organ chambers) das artérias submetidas a infusão de cardioplegia cristalóide a altas pressões (136.66 \pm $39,32 \mathrm{mmHg}$ ) em um curto período de tempo (2 a 3 minutos) (organ chambers), foi uniformemente normal quanto à função endotelial, avaliada pela sua capacidade de produção de óxido nitrico, quando estimulada por agonistas dos passos conhecidos da cascata da produção deste mediador gasoso. Este achado permite as seguintes considerações especulativas:

a) O barotrauma produzido pela infusão de cardioplegia cristalóide a altas pressões ocorrería apenas em circulações coronarianas previamente doentes? Para isso estudos sobre os efeitos das pressões de infusão poderão ser realizadós após submeter o coração a isquemia global seguida de reperfusão, ou em animais com aterosclerose.

b) Uma vez que as infusões duraram de 2 a 3 minutos, sería o barotrauma coronariano um fenômeno dependente do tempo de infusão? Para isso poderão ser realizadas observações em coronárias submetidas a infusões cardioplégicas contínuas durante tempos prolongados variados (30, 60 e 90 minutos).

c) Para que ocorra o barotrauma seriam necessários níveis mais elevados de potássio? Para isso poderão ser realizados experimentos aumentando a concentração de potássio da solução cardioplégica de $16 \mathrm{mEq} / \mathrm{l}$ para, por exemplo, $46 \mathrm{mEq} / \mathrm{l}$.

d) Questionar a existência do fenômeno do barotrauma coronariano produzido pela infusão de soluções cardioplégicas pelo menos nas condições experimentais utilizadas.

e) A metodologia empregada estuda apenas as reatividade vascular de artérias coronarianas epicárdicas. Estas artérias seríam menos sensíveis aos efeitos da pressão de infusão da cardioplegia do que a microcirculação coronariana? Os estudos usando a preparação de Langendorff para coração isolado sugere que isso possa ocorrer. Porém, os estudos utilizando esta metodologia apresentam muitas dúvidas, relacionadas, principalmente, com a garantía de uniformidade dos experimentos.

f) Seria a circulação coronária do cão menos sensível a altas pressões do que a do homem?

Em conclusão, os presentes experimentos sugerem que a infusão de cardioplegia cristalóide, moderadamente hipocalêmica, a altas pressões em um tempo de 2 a 3 minutos, não interfere com a 
produção de EDRF/NO pelo endotélio de coronárias epicárdicas do cão. Esta observação experimental permite a série de considerações especulativas acima mencionadas. Em outras palavras, estes dados refletem uma incerteza sobre uma possível lesão endotelial causada pela infusão de solução cardioplégica. Experimentos adicionais utilizando-se ensaios biológicos de veias safenas, mostraram que distensões a altas pressões não alteraram a função endotelial quanto à produção de EDRF/NO (Schaff: dados não publicados). Portanto, a lesão por barotrauma de células endoteliais é difícil de ser documentada do ponto de vista funcional. Existem, certamente, evidências histológicas do barotrauma vascular, mas as células endoteliais remanescentes apresentam uma adequada função.

\section{RBCCV 44205-298}

Évora P R B, Pearson P J, Oeltjen M, Discigil B, Schaff H V - Crystalloid cardioplegia, barotrauma and endothelium function: experimental considerations. Rev Bras Cir Cardiovasc 1996;11 (2): 115-22.

ABSTRACT: Experiments were performed in "organ chambers" to investigate if high pressures infusions of crystalloid cardioplegia effect the endothelium function of epicardic canine coronary arteries. These experiments did not show any alterations at level of receptors (dose-response curves to $A C H$ and $A D P$ ); signal transduction/G-proteins (dose-response curve to sodium fluoride); intracellular mechanisms of the EDRF/NO release (dose-response curves to phospholipase $\mathrm{C}$ and calcium ionophores $\mathrm{A23187}$ ). The smooth muscular relaxant function (dose-response curves to sodium nitroprusside and isoproterenol) and contarctions (doseresponse curves to $\mathrm{KCl}$ and PGF2alpha) were also preserved. These experimental observations allow the following speculative considerations: a) Should barotrauma be a phenomenon present only in damaged coronary circulation? b) All infusion were performed in no more than two or three minutes. Is cardioplegia barotrauma a phenomenon time-dependente? c) High levels of potassium could be associated with barotrauma. d) Cardioplegia barotrauma is a fancy, at least in our experimental conditions? e) Experiments in "organ chambers" study only epicardic arteries. Could barotrauma damage the microcirculation? f) The canine coronary circulation is less affeccted by high pressure than human coronaries? These data are suggestive that crystalloid moderately hyperkalemic infusions at high pressures for two or three minutes, do not impair the endothelium release of EDRF/NO of canine epicardic coronary arteries.

DESCRIPTORS: Heart arrest, induced, methods. Barotrauma. Endothelium vascular, drug effects. Pericardium, drug effects. Coronary vessels, drug effects. Cardioplegic solutions, pharmacology.

\section{REFERÊNCIAS BIBLIOGRÁFICAS}

1 Chiavarelli R, Macchiarelli G, Familiari G et al. Ultrastructural changes of coronary artery endothelium induced by cardioplegic solutions. Thorac Cardiovasc Surgeon 1989; 37: 151-7.

2 Harjula A, Mattila S, Myllarniemi $\mathrm{H}$ et al. - Effects of synthetic blood and crystalloid cardioplegia solutions on coronary endothelium: an experimental scanning electron microscopic study. J Surg Res 1985; 39: 405-12.

3 Molina J E, Galliani C A, Einzig S, Bianco R, Rasmussen T, Clack R - Physical and mechanical effects of cardioplegic injection on flow distribution and myocardial damage in hearts with normal coronary arteries. J Thorac Cardiovasc Surg 1989; 97: 870-7.
Kontos H A \& Wei E P - Reversal of acetylcholineinduced cerebral vasodilation in acute hypertension Microvasc Res 1985; 29: 231-2.

5 Furchgott R F \& Zawadski J V - The obligatory role of endothelial cells in the relaxation of arterial smooth muscle by acetylcholine. Nature 1980; 228: 373-6.

6 Furchgott R F - Studies on relaxation of rabbit aorta by sodium nitrite: the basis for the proposal that the acid-activated inhibitory factor from bovine retractor penis is inorganic nitrite and the endothelium-derived relaxing factor is nitric oxide. In: Vanhoutte PM ed. Mechanisms of vasodilatation, New York, Raven Press 1988. Vol IV, p. 401-14.

7 Ignarro L J, Byrns R E, Wood K S - Biochemical and pharmacological properties of EDRF and its similarity to nitric oxide radical. In: Vanhoutte PM (ed.) 
Mechanisms of vasodilatation, New York, Raven Press 1988; Vol IV, p. 427-35.

8 Palmer R M J, Ashton D S, Moncada S - Vascular endothelial cells synthesize nitric oxide from Larginine. Nature 1988; 333: 664-6.

9 Carpentier S, Murawsky M, Carpentier A - Cytotoxicity of cardioplegic solutions: evaluation by tissue culture. Circulation 1981; 64 (Supl 2): 90-5.

10 Solberg S, Larsen T, Lindal S, Prydz P, Jorgensen L, Sorlie D - The effects of two different crystalloid cardioplegic solutions on cultured human endothelial cells. J Cardiovasc Surg 1989; 30: 669-74.

11 Saldanha C \& Hearse D J - Coronary vascular responsiveness to 5 -hydroxytryptamine before and after infusion of hyperkalemic crystalloid cardioplegic solution in the rat heart. $J$ Thorac Cardiovasc. Surg 1989; 98: 783-7.

12 Evora P R B, Pearson P J, Schaff H V - Crystaloid cardioplegia and hypothermia do not impair endothelium-dependent relaxation or damage vascular smooth muscle of epicardial coronary arteries. J Thorac Cardiovasc Surg. 1992; 104: 1365-74.

13 Evora P R B - O impacto científico da descoberta do óxido nítrico como vasodilatador e antitrombótico endógeno (Editorial). Arq Bras Cardiol 1993; 61: 3-5.

14 Evora P R B, Peărson P J, Schaff H V - Impaired endothelium-dependent relaxation to sodium fluoride following coronary reperfusion: evidence for G-protein dysfunction. Circulation 1991; 84 (Supl 2): 275.

15 Flavahan N A \& Vanhoutte P M - Pertussis toxin inhibits endothelium-dependent relaxations evoked by fluoride. Eur J Pharmacol 1990; 178: 121-4.

16 De Nucci G, Gryglewski R J, Warner T D, Vane J R Receptor-médiated release of endothelium-derived relaxing factor and prostacyclin from bovine aortic endothelial cells is coupled. Proc Natl Acad Sci (USA) 1988; 85: 2334-8. 